\title{
Variability in the diagnosis of dysplasia in ulcerative colitis by dynamic telepathology
}

\author{
ROBERT D. ODZE ${ }^{1}$, JOHN E. TOMASZEWSKI ${ }^{2}$, EMMA E. FURTH ${ }^{2}$, MICHAEL D. FELDMAN $^{2}$, \\ RAIHANATOU DIALLO ${ }^{3}$, CHRISTOPHER POREMBA $^{4}$, INGRID BECKER $^{5}$, HEINZ HOEFLER $^{4}$, \\ JOHN R. GOLDBLUM ${ }^{6}$, LISA A. RYBICKI ${ }^{7}$, NADA ALSAIGH ${ }^{8}$ and FRANZ FOGT ${ }^{2}$
}

\begin{abstract}
Departments of Pathology, ${ }^{1}$ Brigham and Women's Hospital, Boston, MA; ${ }^{2}$ University of Pennsylvania Presbyterian Medical Center, Philadelphia, PA, USA; ${ }^{3}$ University of Muenster; ${ }^{4}$ University of Duesseldorf; ${ }^{5}$ Technische University Munich, Germany; ${ }^{6}$ Cleveland Clinic; ${ }^{7}$ University of Cincinnati, OH; ${ }^{8}$ Dianon Systems, Stratford, CT, USA
\end{abstract}

Received May 9, 2006; Accepted July 28, 2006

\begin{abstract}
Telepathology (TP) is the practice of evaluating pathology cases by the digital transmission of diagnostic slides as either static pictures (static TP) or by a continuous flow of pictures from a robotic microscopy (dynamic TP). The diagnostic efficacy of dynamic TP-based consultation services has not been widely tested. Dysplasia arising in association with chronic ulcerative colitis (CUC) is, at present, the most important marker for an increased risk of malignancy in patients with this disease. However, the diagnosis of dysplasia suffers from a significant degree of intra- and interobserver variability which usually necessitates a second opinion prior to definitive treatment. Thus, it is often necessary to obtain expert consultation of potential dysplasia cases by dedicated gastrointestinal pathologists. The aim of this study was to evaluate the utility and interobserver variability of diagnosing dysplasia in CUC with the use of dynamic TP. Dynamic TP was used to evaluate digitalized images of 38 CUC cases with areas considered negative, indefinite, or positive for dysplasia (low or high grade) independently by seven pathologists. Subsequently, all cases were graded by each of the pathologists by light microscopic examination of the H\&E-stained glass slides. The degree of intra- and interobserver variability was determined by Kappa statistics. Overall, there was a poor degree of interobserver agreement $(K=0.32)$ among the seven pathologists after analysis of the cases by dynamic TP. The poorest level of agreement was in the indefinite and lowgrade dysplasia categories, whereas the highest level was in the negative and high-grade dysplasia categories. Grouping together several diagnostic categories (for instance: Indefinite
\end{abstract}

Correspondence to: Dr Franz Fogt, Pathology and Laboratory Medicine, University of Pennsylvania, Presbyterian Medical Center, 39th and Market Streets, Philadelphia, PA 19104, USA

E-mail: franz.fogt@uphs.upenn.edu

Key words: ulcerative colitis, telepathology and low, or low- and high-grade dysplasia) had no significant effect on the level of agreement. The degree of variability in interpretation of cases by microscopic slide analysis was similar $(\mathrm{K}=0.35)$. After reviewing all the cases by microscopic analysis of the glass slides, the diagnosis was changed in $51 \%$ of the observations; in the majority of these (61\%), the grade of dysplasia was decreased. In summary, the use of dynamic TP for consultation in CUC-associated dysplasia has a poor level of interobserver agreement, but does not differ significantly from that obtained by the evaluation of the cases by microscopic slide analysis. Diagnoses rendered by dynamic TP tend to be of a higher grade compared to that obtained by microscopic slide analysis. Thus, although dynamic TP may be used for the consultation of CUC dysplasia cases, more specific criteria are needed in the general categorization of dysplasia in CUC.

\section{Introduction}

Telepathology (TP) is the process of evaluating pathology cases by digitally transmitted pictures on a computerized display screen (1-3). There are two main types of TP: Static (stationary) and dynamic (robotic). Whereas static image TP involves the transmission of still pictures from one pathologist to another at a remote site, dynamic TP incorporates the ability of the receiving pathologists to have real-time interaction simply by controlling the movement of the pathology slide in the microscopic state. The main advantage of the robotic units is that real-time interaction is possible and decreases the likelihood of missing important elements on the pathology slides (1).

Several studies have evaluated the diagnostic accuracy of static, dynamic and various hybrid forms of TP (1-8). In many of these studies, the efficacy of evaluating cases by digital images proved to be quite reliable. For instance, some studies have shown that TP can be applied successfully to intraoperative consultation services $(7,9)$. Nevertheless, diagnostic difficulties and discrepancies are encountered more often when evaluating pathological entities in diagnostically difficult areas of pathology. This applies, in particular, to areas of pathology where diagnoses are based 
on accurate and specific architectural and/or cytological abnormalities, and where interobserver agreement with the use of conventional microscopic examination is quite high. For instance, dysplasia that arises in association with chronic ulcerative colitis (CUC) is the most important marker for an increased risk of malignancy in this condition, and is critical in guiding patient management (10). Since histological distinction between the various categories of dysplasia is often subtle, and suffers from a significant degree of intraand interobserver variability (11), it is usually necessary to obtain confirmation of the diagnosis prior to treatment (10-13). In fact, the American College of Gastroenterology recommends that at least one experienced gastrointestinal (GI) pathologist should confirm any potential diagnosis of dysplasia in CUC prior to definitive treatment. This is often difficult since most general practice institutions do not have a dedicated GI pathologist.

We have previously shown in a static TP study of dysplasia in CUC that the diagnostic efficacy is similar to that found in microscopic slide analysis but that static TP suffers from limitations due to the bias of field selections, the quality of still pictures, and the inability to visualize surrounding non-lesional tissue. Therefore, we performed this study to determine whether dynamic (robotic) TP has a higher level of interobserver agreement with regard to diagnosing dysplasia in CUC, since this method of analyzing tissue has been reported to decrease the prevalence of these previously mentioned technical limitations.

\section{Materials and methods}

Analysis of digitalized images. Seven pathologists with special interest in TP, were involved in this study. One of these pathologists is a dedicated GI pathologist, the others have expertise in different areas of surgical pathology other than GI pathology. In total, 38 cases of CUC-related dysplasia (low and high grade) or cases considered 'negative' or 'indefinite for dysplasia' were retrieved from archival mucosal biopsy pathology material and used for this study. The initial 'working' diagnosis was rendered by another GI pathologist, who served as the reference pathologist, by analysis of the glass slides, using previously published criteria (11). This reference pathologist established a diagnosis of negative for dysplasia in 11 cases, indefinite in 5, low-grade dysplasia (LGD) in 12 and high-grade dysplasia (HGD) in 10 cases. Each of the reviewing pathologists were then asked to evaluate all the cases separately with the use of a TP instrument by using a remote control for moving, focusing and changing the digital picture on the computer screen in order to render a dysplasia grade. The Apollo Telepath system (Apollo Telemedicine, Falls Church, VA, USA) was used. This is a dynamic TP system based on a Sony 3CCD camera (Sony, Cologne, Germany) and online data transmission via a glass fibre network and a cooperative project TP system between Nikon and ZEM (ZEM Technology, Hoogblokland, The Netherlands). Each pathologist was also asked to comment on the difficulties encountered during the analysis of the digitalized images using this dynamic TP process. All the results, and comments, were submitted to the reference pathologist for statistical analysis.
Analysis of microscopic slides. In the second part of the study, that was performed at least 3 months after completion of the digital evaluation of the slides, all glass slides were renumbered and an additional 12 colonic mucosal biopsies of similar diagnostic categories were added to the study set to avoid recall bias. Each of the seven reviewing pathologists were then asked to re-evaluate all the cases (total: 50) by light microscopic examination of the H\&E-stained glass slides and to, once again, select the most appropriate dysplasia diagnostic category (negative, indefinite, LGD, HGD). However, the diagnostic results of the 12 additional cases were not used in the analysis of the results.

Statistics. The Kappa statistics method was used to assess interobserver and intraobserver variability separately based on the analysis of the digitalized images or by the microscopic slides among the seven reviewing pathologists. The results were compared between the two methods using the $z$ test. The Kappa statistics was also used to assess interobserver variability between the digitalized images and microscopic slides separately for each of the four reviewing pathologists. In addition, multiple different categorizations of the dysplasia grade were analyzed. i) Negative vs indefinite vs LGD vs HGD, ii) negative/ indefinite vs LGD vs HGD, iii) negative vs indefinite/LGD vs HGD, iv) negative vs indefinite vs LGD/HGD, v) negative/indefinite vs LGD/ HGD, vi) negative/indefinite/ LGD vs HGD. Kappa measures agreement beyond that which is expected by chance alone. Values $>0.75$ indicate excellent agreement beyond chance, values between 0.40 and 0.75 indicate fair to good agreement beyond chance, and values of $<0.40$ indicate poor agreement (15). The results of the Kappa analysis are summarized as the Kappa value, 95\% confidence interval, and the P-value from the $z$ test, which determines whether agreement is better than that which is expected by chance alone. Individual Kappas indicate agreement within individual diagnostic categories, whereas the overall Kappa is a weighted average of the individual Kappa values. All analyses were conducted using SAS software (SAS Institute, Cary, NC, USA): A P-value of $<0.05$ was the criterion for statistical significance.

\section{Results}

Interobserver variability of analysis of digitalized images by dynamic telepathology. Table I provides a summary of the degree of agreement in the interpretation of the cases (categorization no. i) by TP. Overall, there was a poor level of interobserver agreement $(\mathrm{K}=0.32)$ among the 7 reviewing pathologists after analysis of the cases by dynamic TP. The poorest level of agreement was in the indefinite $(K=0.01)$ and low-grade dysplasia $(K=0.26)$ categories. Negative for dysplasia and high-grade dysplasia showed a better ('fair') agreement. Grouping together several diagnostic categories (for instance, analysis of the cases by categorization nos. ii-vi; see Materials and methods) had no significant effect on the level of agreement. For instance, when the diagnostic grades of negative for dysplasia and indefinite for dysplasia were combined as a single diagnostic entity, or when LGD and HGD were combined as a single entity, the degree of agreement remained poor ( $\mathrm{K}=0.33$ and 0.36 , respectively). 
Table I. Overall Kappa indices for the interobserver agreement.

\begin{tabular}{|c|c|c|c|c|c|}
\hline Method & $\begin{array}{l}\text { Overall } \\
\text { Kappa }\end{array}$ & $\begin{array}{c}\text { Individual } \\
\text { Kappa }\end{array}$ & P-value & $95 \% \mathrm{CI}$ & Interpretation \\
\hline Telepathology & 0.32 & & $<0.001$ & $0.28-0.36$ & Poor \\
\hline Negative & & 0.43 & $<0.001$ & $0.36-0.50$ & Fair \\
\hline Indefinite & & 0.01 & 0.71 & $-0.06-0.08$ & Poor \\
\hline LGD & & 0.26 & $<0.001$ & $0.19-0.33$ & Poor \\
\hline HGD & & 0.46 & $<0.001$ & $0.39-0.53$ & Fair \\
\hline Microscopic slides & 0.35 & & $<0.001$ & $0.31-0.39$ & Poor \\
\hline Negative & & 0.47 & $<0.001$ & $0.40-0.54$ & Fair \\
\hline Indefinite & & -0.05 & $<0.18$ & $-0.12-0.02$ & Poor \\
\hline LGD & & 0.34 & $<0.001$ & $0.27-0.40$ & Poor \\
\hline HGD & & 0.38 & $<0.001$ & $0.31-0.45$ & Poor \\
\hline
\end{tabular}

${ }^{a}$ Agreement beyond chance; poor, $\mathrm{K}<0.40$; fair to good, $\mathrm{K} \geq 0.40-\leq 0.75$; negative, negative for dysplasia; indefinite, indefinite for dysplasia; LGD, low-grade dysplasia; HGD, high-grade dysplasia.

Interobserver variability of microscopic slide analysis. The lower part of Table I provides a summary of the data from the microscopic slide analysis (categorization no. i). The degree of interobserver agreement in the interpretation of the microscopic slides was similar to that obtained by dynamic TP $(\mathrm{K}=0.35)(\mathrm{P}>0.05)$. Similar to dynamic TP, the level of agreement was highest for the negative and HGD cases, and lowest for the indefinite and LGD cases. However, in the case of microscopic slide analysis, combining the cases of negative for dysplasia and indefinite for dysplasia into a single diagnostic category (categorization no. ii) increased the level of agreement to fair (0.40), but this was not statistically significant $(\mathrm{P}>0.05)$.

Intraobserver analysis of cases evaluated by dynamic telepathology vs microscopic slide analysis. Table II outlines the level of intraobserver agreement between the analysis of the cases by dynamic TP vs microscopic slides (categorization no. i). The overall level of intraobserver agreement was poor ( $\mathrm{K}=0.50$, range $0.28-0.64)$. However, 4 of the 7 pathologists showed 'good' agreement ( 4 cases with $\mathrm{K}>0.58$ ). For 4 of the 7 pathologists, the highest level of intraobserver agreement occurred in the negative for dysplasia and HGD categories. Grouping together cases considered negative for dysplasia and indefinite for dysplasia (categorization no. ii) resulted in a slight increase in intraobserver agreement $(\mathrm{K}=0.59)$, but this was not statistically significant.

Tables III and IV provide more details regarding the diagnostic differences between dynamic TP and the microscopic slides. Table III provides the raw data regarding the diagnostic changes and Table IV provides a summary of the changes. For instance, line 1 in Table III indicates that reviewer no. 1 diagnosed 10 cases as negative for dysplasia by dynamic TP. However, upon light microscopic review of these same 10 cases, 8 cases remained in the negative category and 2 cases were upgraded to HGD. Similarly, line 1 in Table IV summarizes the changes by this same pathologist.
For this individual, 15 of the 38 cases (39\%) were changed to a different histological grade after a review of the cases by light microscopy; 6 were upgraded $(48 \%)$ and 9 were downgraded $(60 \%)$. Overall, 77 of the $152(51 \%)$ cases had a change of diagnosis; 30/77 (39\%) were upgraded and 47/77 $(61 \%)$ were downgraded.

Summary of difficulties reported by pathologists in evaluating cases by dynamic telepathology. A summary of the pathologists' comments regarding the difficulties in the evaluation of the cases of dysplasia in CUC by dynamic TP are indicated in Table $\mathrm{V}$. The most frequently reported difficulty was the problem of scanning large tissue fragments for the presence of areas of interest rather than assessing specific points of interest that are already marked by the pathologist seeking consultation. A similar number of comments were made regarding difficulties with depth perception and inappropriate field selection. Inadequate picture resolution of the digitalized photographs was noted as a disadvantage of TP by some, which led pathologists to have difficulties evaluating nuclear detail and various cellular degenerative changes. Other less frequent comments are also noted in this Table. Interestingly, a significantly lower degree of interobserver agreement was noted between the cases in which problems were noted, compared to cases in which no technical problems were cited $(\mathrm{P}<0.001)$.

\section{Discussion}

Telepathology is the practice of creating and evaluating digitalized microscopic images that are transmitted by telecommunication pathways through computer networks for the purpose of diagnosis, consultation or teaching (1). Telepathology is rapidly gaining widespread acceptance as a viable method of providing consultation pathology services to remote sites. There are two general forms of TP imaging, termed as static and dynamic. In the static type, digitalized 
Table II. Overall Kappa indices for the intraobserver agreement between the readings by dynamic TP and the microscopic slides.

\begin{tabular}{|c|c|c|c|c|c|}
\hline Method & $\begin{array}{l}\text { Overall } \\
\text { Kappa }\end{array}$ & $\begin{array}{c}\text { Individual } \\
\text { Kappa }\end{array}$ & P-value & $95 \% \mathrm{CI}$ & Interpretation $^{\mathrm{a}}$ \\
\hline Pathologist no. 1 & 0.56 & & $<0.001$ & $0.33-0.79$ & Good \\
\hline Negative & & 0.73 & $<0.001$ & $0.41-1.00$ & Excellent \\
\hline Indefinite & & - & - & - & - \\
\hline LGD & & 0.56 & $<0.001$ & $0.24-0.88$ & Good \\
\hline HGD & & 0.42 & $<0.010$ & $0.10-0.73$ & Fair \\
\hline Pathologist no. 2 & 0.41 & & $<0.001$ & $0.18-0.63$ & Fair \\
\hline Negative & & 0.51 & $<0.002$ & $0.19-0.83$ & Fair \\
\hline Indefinite & & -0.01 & 0.93 & $-0.33-0.30$ & Poor \\
\hline LGD & & 0.29 & 0.07 & $-0.03-0.61$ & Poor \\
\hline HGD & & 0.46 & $<0.005$ & $0.14-0.78$ & Fair \\
\hline Pathologist no. 3 & 0.58 & & $<0.001$ & $0.37-0.78$ & Good \\
\hline Negative & & 0.47 & $<0.004$ & $0.15-0.79$ & Fair \\
\hline Indefinite & & -0.07 & 0.66 & $-0.39-0.25$ & Poor \\
\hline LGD & & 0.68 & $<0.001$ & $0.36-0.99$ & Good \\
\hline HGD & & 0.74 & $<0.001$ & $0.43-1.00$ & Excellent \\
\hline Pathologist no. 4 & 0.64 & & $<0.001$ & $0.43-0.85$ & Good \\
\hline Negative & & 0.56 & 0.001 & $0.25-0.88$ & Good \\
\hline Indefinite & & -0.04 & 0.80 & $-0.36-0.28$ & Poor \\
\hline LGD & & 0.73 & 0.001 & $0.42-1.00$ & Excellent \\
\hline HGD & & 0.74 & 0.001 & $0.41-1.00$ & Excellent \\
\hline Pathologist no. 5 & 0.28 & & $<0.006$ & $0.08-0.48$ & Poor \\
\hline Negative & & 0.41 & $<0.012$ & $0.09-0.73$ & Fair \\
\hline Indefinite & & 0.24 & 0.13 & $-0.07-0.56$ & Poor \\
\hline LGD & & 0.21 & 0.20 & $-0.11-0.53$ & Poor \\
\hline HGD & & 0.21 & $<0.19$ & $-0.10-0.53$ & Poor \\
\hline Pathologist no. 6 & 0.61 & & $<0.001$ & $0.42-0.81$ & Good \\
\hline Negative & & 0.68 & $<0.001$ & $0.37-1.00$ & Good \\
\hline Indefinite & & 0.26 & 0.11 & $-0.06-0.57$ & Poor \\
\hline LGD & & 0.60 & $<0.001$ & $0.29-0.92$ & Good \\
\hline HGD & & 0.77 & $<0.001$ & $0.45-1.00$ & Excellent \\
\hline Pathologist no. 7 & 0.42 & & $<0.001$ & $0.20-0.65$ & Fair \\
\hline Negative & & 0.47 & 0.004 & $0.15-0.79$ & Fair \\
\hline Indefinite & & -0.09 & 0.60 & $-0.40-0.23$ & Poor \\
\hline LGD & & 0.56 & 0.001 & $0.25-0.88$ & Good \\
\hline HGD & & 0.36 & 0.027 & $0.04-0.68$ & Poor \\
\hline
\end{tabular}

${ }^{a}$ Agreement beyond chance; poor, $\mathrm{K}<0.40$; fair to good, $\mathrm{K} \geq 0.40-\leq 0.75$; excellent, $\mathrm{K}>0.75$; negative, negative for dysplasia; indefinite, indefinite for dysplasia; LGD, low-grade dysplasia; HGD, high-grade dysplasia.

images of microscopic slides are obtained and transmitted to a referral telepathologist who then views them sequentially on a computerized video monitor. In contrast, dynamic TP involves viewing live video images and microscopic slides that are transmitted and visualized in real-time (1-3). There have been only a few studies that have evaluated the efficacy of dynamic-based TP, and none regarding the use of this system for the evaluation of GI biopsy specimens. Therefore, this study was performed to determine the efficacy of dynamic TP in an area of GI pathology that is known to be particularly susceptible to a high level of interobserver variability, and one in which diagnoses are often based on subtle and difficult cytological and architectural patterns of injury. For instance, in CUC, treatment considerations are 
Table III. Results of telepathology vs histological analysis of the microscopic slides.

\begin{tabular}{|c|c|c|c|c|c|}
\hline \multirow[b]{2}{*}{ Reviewer } & \multirow[b]{2}{*}{$\begin{array}{c}\text { Tele- } \\
\text { pathology }\end{array}$} & \multicolumn{4}{|c|}{ Histology } \\
\hline & & Negative & Indefinite & LGD & HGD \\
\hline \multirow[t]{4}{*}{1} & Negative & 8 & 0 & 0 & 2 \\
\hline & Indefinite & 0 & 0 & 0 & 0 \\
\hline & LGD & 1 & 0 & 11 & 5 \\
\hline & HGD & 1 & 0 & 2 & 8 \\
\hline \multirow[t]{4}{*}{2} & Negative & 8 & 0 & 1 & 1 \\
\hline & Indefinite & 0 & 0 & 1 & 0 \\
\hline & LGD & 5 & 0 & 9 & 4 \\
\hline & HGD & 1 & 0 & 2 & 6 \\
\hline \multirow[t]{4}{*}{3} & Negative & 5 & 0 & 0 & 2 \\
\hline & Indefinite & 3 & 0 & 2 & 0 \\
\hline & LGD & 2 & 0 & 13 & 1 \\
\hline & HGD & 0 & 0 & 1 & 9 \\
\hline \multirow[t]{4}{*}{4} & Negative & 6 & 2 & 2 & 1 \\
\hline & Indefinite & 1 & 0 & 0 & 0 \\
\hline & LGD & 0 & 0 & 15 & 3 \\
\hline & HGD & 0 & 0 & 0 & 8 \\
\hline \multirow[t]{4}{*}{5} & Negative & 6 & 3 & 0 & 0 \\
\hline & Indefinite & 6 & 9 & 1 & 0 \\
\hline & LGD & 0 & 4 & 3 & 0 \\
\hline & HGD & 0 & 0 & 5 & 1 \\
\hline \multirow[t]{4}{*}{6} & Negative & 15 & 1 & 1 & 0 \\
\hline & Indefinite & 3 & 2 & 1 & 0 \\
\hline & LGD & 0 & 1 & 4 & 0 \\
\hline & HGD & 1 & 1 & 1 & 7 \\
\hline \multirow[t]{4}{*}{7} & Negative & 16 & 0 & 2 & 0 \\
\hline & Indefinite & 6 & 0 & 0 & 0 \\
\hline & LGD & 2 & 0 & 8 & 2 \\
\hline & HGD & 0 & 0 & 1 & 1 \\
\hline
\end{tabular}

LGD, low-grade dysplasia; HGD, high-grade dysplasia.

based on the degree of dysplasia $(10,11)$. High-grade dysplasia is generally an indication for a colectomy, whereas negative, indefinite and some low-grade lesions, are normally managed by either regular or increased surveillance, respectively (10). In fact, the American College of Gastroenterology (ACG) strongly recommends that a case considered to represent CUC-related dysplasia should be confirmed by a pathologist with particular expertise in GI prior to definitive treatment. Therefore, the use of a TP system to evaluate and confirm dysplasia cases in CUC would be of great value to the general pathology community, and would serve to decrease the cost, and shorten the turn-around time, of expert consultation.
Table IV. Summary of the disagreement between telepathology and histological analysis of the microscopic slides.

\begin{tabular}{lrrr}
\hline Pathologist & $\begin{array}{c}\text { No. of diagnoses } \\
\text { changed (\%) }\end{array}$ & Higher (\%) & Lower (\%) \\
\hline 1 & $11 / 38(29)$ & $7(64)$ & $4(36)$ \\
2 & $15 / 38(39)$ & $6(40)$ & $9(43)$ \\
3 & $11 / 38(29)$ & $5(45)$ & $6(55)$ \\
4 & $9 / 38(24)$ & $8(89)$ & $1(11)$ \\
5 & $19 / 38(50)$ & $4(21)$ & $15(79)$ \\
6 & $10 / 18(56)$ & $3(30)$ & $7(70)$ \\
7 & $13 / 18(72)$ & $4(31)$ & $9(69)$ \\
Total $1-7$ & $77 / 152(51)$ & $30 / 77(39)$ & $47 / 77(61)$ \\
\hline
\end{tabular}

Table V. Summary of the pathologists' comments regarding the limitations of evaluating the digitalized photographs by dynamic telepathology photographs.

Pathologists' comments

No. of observations $(\mathrm{N}=152)$

$\begin{array}{lr}\text { Inappropriate field selection } & 4 \\ \text { Difficulty with depth perception } & 4 \\ \text { Difficulty in assessing large sections } & 4 \\ \text { vs 'points of interest' } & 3 \\ \text { Slow movement } & 3 \\ \text { Poor nuclear detail } & 2 \\ \text { Poor detail of cellular changes } & \\ \text { (apoptotic figures vs mitoses) } & 2 \\ \text { Poor low power assessment } & \end{array}$

The results of this study showed that there was an overall poor degree of interobserver agreement $(\mathrm{K}=0.32)$ among the seven pathologists in interpreting CUC-related dysplasia cases by dynamic TP. Unfortunately, the level of agreement was not improved by grouping together several different diagnostic categories that would normally have clinical significance in terms of treatment. However, the degree of variability in the interpretation of the same cases by microscopic slide analysis was similar, and serves to reiterate the rather well-known finding that there are limitations in the interpretation of dysplasia in CUC by light microscopy. Hard objective criteria are needed in order to improve the diagnostic accuracy of this condition. This is also highlighted by the fact that our study also showed that the best levels of agreement were obtained for cases which are representative of the two extremes of the continuous spectrum of atypical changes in CUC; negative for dysplasia and high-grade dysplasia. These findings have been observed in other interobserver variability studies in the interpretation of dysplasia in CUC by microscopic slide analysis $(11,13,17)$. 
Another important result of this study is the fact that, overall, $51 \%$ of the TP-derived diagnoses were changed after an analysis of the slides by light microscopy. Of the seven pathologists, the number of cases in which the diagnosis was changed ranged from $24 \%$ to $72 \%$. The majority of revised diagnoses $(61 \%)$ were downgraded to a lower grade of dysplasia. In fact, only two of the reviewing pathologists changed more of their diagnoses to a higher histological grade, compared to the others. The reasons for these findings are unclear. However, in general pathology practice, it is more common for CUC-associated dysplasia diagnoses to be downgraded by expert consultants. This is likely due to the lack of general awareness in the general practice community of the wide range of regenerative changes that can occur in CUC, particularly when active inflammation is present in the biopsy sample.

There has been only one other previously published study that has evaluated the degree of interobserver variability in diagnosing CUC-associated dysplasia by TP (14). In that study, which was performed by some members of our research group, static, electronically transmitted digitalized images of dysplasia cases were evaluated independently by four dedicated GI pathologists. In that study, there was also an overall fair degree of agreement $(\mathrm{K}=0.4)$, with the highest levels of agreement also in the negative and high-grade dysplasia categories. In that study, variability in the interpretation of the glass slides was similar to static TP. However, in contrast to the results of this study, the static TP-based study showed a higher rate of conversion of diagnoses to a higher grade after the analysis of the microscopic slides. The reasons cited for a change of diagnosis in that study were related to limitations regarding field selection, and/or an inability to view the mucosa distant to the atypical focus, both of which are well-known limitations of the static TPbased system.

As mentioned above, of particular significance is the fact that the level of agreement achieved by a review of digitalized images by dynamic TP in this study was only slightly lower than that obtained after a review of the original glass slides by light microscopy. This suggests to us that it is not necessarily the type of technique used to observe the histological sections (i.e. TP vs microscopic slide analysis) that results in the variability in interpretation, but probably the inherent inadequacy of objective and reproducible criteria presently in use for grading dysplasia in CUC. This is a major reason why there has been a recent explosion of interest in finding new, more reproducible and objective, measures of risk of malignancy in CUC, such as aneuploidy, and the evaluation of molecular markers such as p53 (18).

The level of interobserver agreement by analysis of the TP-based images in this study is similar to that obtained in other interobserver variability studies that were based upon the microscopic analysis of glass slides $(11,13,16,17)$. Although the results of these other studies are difficult to compare to ours because of differences in the methods used for statistical comparisons, the levels of agreement in other studies have ranged from Kappa values of 0.29 to 0.58 . For instance, in an interobserver variability study by Dixon et al that included 100 cases and four diagnostic categories (hyperplasia, reactive atypia, low-grade and high-grade), and six participating pathologists, the overall level of agreement among all the pairs of observers was considered only fair $(\mathrm{K}=0.41)$, with a wide range of Kappa values (13). Therefore, we think that although our findings showed that the level of interobserver agreement in the diagnosis of dysplasia in CUC by $\mathrm{TP}$ is, at best, fair, and needs significant improvement, based on the criteria available at this time, we believe that the diagnostic consultation of these cases by dynamic TP is an acceptable alternative to traditional glass slide analysis. This statement is further supported by the results of other studies which have observed a high level of diagnostic accuracy with the use of either static or dynamic-based TP systems utilizing pathology material other than GI mucosal biopsies $(2-5,8,19-22)$. These studies have included the evaluation of inter-operative frozen sections, prostate needle biopsies, and pulmonary and neuropathology cases.

There are many advantages of the dynamic-based TP system in comparison to the static type (1-3). Several studies have shown a higher level of diagnostic accuracy with the dynamic system in comparison to the static system $(4,23)$. Furthermore, with the dynamic TP system, the consultation may serve as the TP system operator and can control, freely, all microscopic functions, including stage movements. This simulates the physical and intellectual process that ordinarily takes place in a light microscopic session, and enables the telepathologist to view all sections on all glass slides in their entirety. This serves to eliminate the problem of inappropriate field selection which is common with the static TP-based system $(1,2,18)$. Furthermore, dynamic TP has the ability to view an unlimited number of images per case, has a much lower transmission time per image, and average time to diagnosis, compared to static-based TP systems (1). However, both types of systems have shown difficulty with regard to discerning fine cellular details, such as nuclear structure, which can make distinguishing the various grades of dysplasia in any organ system difficult. It is likely that the adequate visualization of nuclear structure may require higher video resolution, which is currently being developed in several types of virtual video-imaging systems $(22,25)$.

In our study, the potential limitations of dynamic TP for assessing dysplasia in CUC were summarized by the participating pathologists (Table V). The most common complaints were with regard to difficulty with depth perception, and difficulty in assessing large sections vs targeting directly the histological points of interest. Some pathologists also commented that the slow movement of the dynamic TP system curtailed their ability to establish a correct diagnosis in some cases. As mentioned above, poor detail of cellular changes, such as nuclear changes, apoptotic figures and mitoses, also contributed to the limitations of the dynamic TP system in this study. However, overall, the pathologists in this study had relatively few problems in using and interpreting cases by dynamic TP. Thus, it is reasonable to assume that as the technology related to TP advances with time, users will become more knowledgeable and better acquainted with the particular operating characteristics of the system and potential sources of error will likely be minimized.

In summary, this study suggests that dynamic TP-based consultation services may be used cautiously for evaluating 
CUC-associated dysplasia. Although the degree of interobserver variability was poor, it was comparable to that in other studies that utilized light microscopic examination of slides, and other studies that used static TP-based systems. Pathologists using this system should be aware that there is a tendency to over-diagnose dysplasia, in comparison to the results obtained by microscopic analysis of glass slides. Better, more precise, criteria for dysplasia in CUC are needed to increase the degree of agreement among observers.

\section{References}

1. Weinstein RS, Bloom KJ and Rozek LS: Telepathology and the networking of pathology diagnostic services. Arch Pathol Lab Med 111: 646-652, 1987.

2. Weinstein RS, Bhattacharyya AK, Graham AR, et al: Telepathology: a ten-year progress report. Hum Pathol 28: 1-7, 1997.

3. Cross SS, Dennis T and Start RD: Telepathology: current status and future prospects in diagnostic histopathology. Histopathology 41: 91-109, 2002

4. Dunn BE, Almagro UA, Choi $\mathrm{H}$, et al: Dynamic-robotic telepathology: department of Veterans Affairs feasibility study. Hum Pathol 28: 8-12, 1997.

5. Weinstein $\mathrm{MH}$ and Epstein JI: Telepathology diagnosis of prostate needle biopsies. Hum Pathol 28: 22-29, 1997.

6. Nordrum I, Engum B, Rinde E, et al: Remote frozen section service: telepathology project in Northern Norway. Hum Pathol 22: 514-518, 1991

7. Halliday BE, Bhattacharyya AK, Graham AR, et al: Diagnostic accuracy of an international static-imaging telepathology consultation service. Hum Pathol 28: 17-21, 1997.

8. Eusebi V, Foschini L, Erde S, et al: Transcontinental consults in surgical pathology via the Internet. Hum Pathol 28: 13-16, 1997.

9. Deminichelis F, Barbareschi M, Boi S, et al: Robotic telepathology for intraoperative remote diagnosis using a stillimaging-based system. Am J Clin Pathol 116: 744-752, 2001

10. Goldman H: Significance and detection of dysplasia in chronic colitis. Cancer 78: 261-263, 1996.

11. Riddell RH, Goldman H, Ransohoff DF, et al: Dysplasia in inflammatory bowel disease: standardized classification with provisional clinical applications. Hum Pathol 14: 931-968, 1983.
12. Riddell RH: Implications of a diagnosis of dysplasia in ulcerative colitis. J Gastroenterol 30 (suppl VIII): 25-29, 1995.

13. Dixon MF, Brown LJR, Gilmour HM, et al: Observer variation in the assessment of dysplasia in ulcerative colitis. Histopathology 13: 385-397, 1988

14. Odze RD, Goldblum J, Noffsinger A, et al: Interobserver variability in the interpretation of ulcerative colitis-associated dysplasia by telepathology. Mod Pathol 15: 379-386, 2000.

15. Fleiss J: Statistical methods for rates and proportions. 2nd edition. Wiley, New York, pp191-230, 1981.

16. Melville DM, Jass JR, Morson BC, et al: Observer study on the grading of dysplasia in ulcerative colitis; comparison with clinical outcome. Hum Pathol 20: 1008-1014, 1990.

17. Dundas SAC, Kay R, Beck S, et al: Can histopathologists reliably assess dysplasia in chronic inflammatory bowel disease? J Clin Pathol 40: 1282, 1987.

18. Odze RD: Diagnostic problems and advances in inflammatory bowel disease. Mod Pathol 16: 347-358, 2003.

19. Pan C-C, Lian W-Y, Huang C-W, et al: Diagnosing minimal adenocarcinoma on prostate needle biopsy by real-time dynamic telepathology through the internet evaluation of an economic technology for remote consultation. Hum Pathol 33: 242-246, 2002.

20. Leong FJW-M, Nicholson AG and McGee OD: Robotic telepathology: efficacy and usability in pulmonary pathology. $\mathrm{J}$ Pathol 197: 211-217, 2002.

21. Szymas J, Wolf G, Papierz W, et al: Online internet-based robotic telepathology in the diagnosis of neuro-oncology cases: a teleneuropathology feasibility study. Hum Pathol 32: 1304-1308, 2001.

22. Singson RPC, Natarajan S, Greenson J, et al: Virtual microscopy and the internet as telepathology consultation tools. A study of gastrointestinal biopsy specimens. Am J Clin Pathol 111: 792-795, 1999

23. Shimasoto Y, Yagi Y, Yamagishi K, et al: Experience and present status of telepathology in National Cancer Center Hospital, Tokyo. Zentralbl Pathol 138: 413-417, 1992.

24. Krupinski EA, Weinstein RS, Bloom KJ, et al: Progress in telepathology: system implementation and testing. Adv Pathol Lab Med 6: 63-87, 1993.

25. Dee FR, Lehman JM, Consoer D, et al: Implementation of virtual microscope slides in the annual pathobiology of cancer workshop laboratory. Hum Pathol 34: 430-436, 2003. 